\title{
Millimeter Wave Spectroscopy of Ne-CO
}

\author{
D. A. Roth ${ }^{\mathrm{a}}$, I. Pak ${ }^{\mathrm{a}, \mathrm{b}}$, L. A. Surin ${ }^{\mathrm{a}, \mathrm{b}}$, B. S. Dumesh ${ }^{\mathrm{b}}$, and G. Winnewisser ${ }^{\mathrm{a}}$ \\ ${ }^{a}$ I. Physikalisches Institut, Universität zu Köln, Zülpicher Str. 77, 50937 Köln, Germany \\ ${ }^{b}$ Institute of Spectroscopy, Russian Academy of Sciences, 142092 Troitsk, Moscow Region, Russia \\ Reprint requests to Prof. G. W.; E-mail: winnewisser@ph1.uni-koeln.de
}

Z. Naturforsch. 55 a, 754-758 (2000); received June 10, 2000

\begin{abstract}
The pure rotational $b$-type spectrum of the van der Waals complex $\mathrm{Ne}-\mathrm{CO}$ has been measured using a pulsed jet, intracavity millimeter wave spectrometer. The millimeter wave generation is based on the OROTRON principle. The high sensitivity of the spectrometer allowed measurements of $R(J), K=1 \leftarrow 0$ transitions between 108 and $150 \mathrm{GHz}$ of the $\mathrm{Ne}$ isotopomers ${ }^{20} \mathrm{Ne}-\mathrm{CO}$ and ${ }^{22} \mathrm{Ne}-\mathrm{CO}$. This new millimeter wave data set together with the microwave data in the literature, i.e. $a$-type microwave transitions, yield in a fit to an asymmetric rotor a reliable set of ground state constants. These are for ${ }^{20} \mathrm{Ne}-\mathrm{CO}: A=107127.021(14) \mathrm{MHz}, B=3479.6597(95) \mathrm{MHz}$, and $C=3039.5387(93) \mathrm{MHz}$. For both ${ }^{20} \mathrm{Ne}-\mathrm{CO}$ and ${ }^{22} \mathrm{Ne}-\mathrm{CO}$, a global fit to a near-symmetric rotor was performed, taking into account the infrared and microwave transition frequencies from the literature and the millimeter wave measurements of the present work.
\end{abstract}

Key words: Van der Waals Complexes; Spectroscopy; Ne-Co; OROTRON.

\section{Introduction}

Among the rare gas-CO van der Waals complexes, $\mathrm{Ne}-\mathrm{CO}$ is one of the less extensively investigated ones, although several studies, both experimental and theoretical, have been recently performed. First evidence of the existence of $\mathrm{Ne}-\mathrm{CO}$ came from the measurements of the infrared spectrum of the $\mathrm{CO}$ subunit's stretching fundamental at $2140 \mathrm{~cm}^{-1}$ [1]. The spectra of ${ }^{20} \mathrm{Ne}-\mathrm{CO}$ and ${ }^{22} \mathrm{Ne}-\mathrm{CO}$ were recorded using two complementary techniques: a cold static gas cell in conjunction with a Fourier transform (FT) spectrometer and a tunable diode laser spectrometer with a pulsed supersonic jet. The pulsed beam FT microwave technique was used in order to investigate the lowest pure rotational $a$-type transitions of several isotopic modifications of $\mathrm{Ne}-\mathrm{CO}$ in the frequency range of 6.0 to $26 \mathrm{GHz}$ [2]. The main isotopic modification ${ }^{20} \mathrm{Ne}-\mathrm{CO}$ was studied in more detail by using a combination of a diode laser spectrometer with a long-path low-temperature absorption cell [3]. In a recent communication [4], we reported the detection of millimeter wave transitions arising from the $K=1 \leftarrow 0$ subband of ${ }^{20} \mathrm{Ne}-\mathrm{CO},{ }^{21} \mathrm{Ne}-\mathrm{CO}$, and ${ }^{22} \mathrm{Ne}-\mathrm{CO}$. The accurate experimental determination of the molecular parameters of the $\mathrm{Ne}-\mathrm{CO}$ complex provides the most sensitive test for the recent ab initio potential energy surfaces $[5,6]$.

In the present paper we complete these earlier measurements by reporting $17 R$-branch transitions for the $\mathrm{Ne}$ isotopic species ${ }^{20} \mathrm{Ne}-\mathrm{CO}$ and ${ }^{22} \mathrm{Ne}-\mathrm{CO}$. They belong to the $R$-branch with $J \leq 10, v=0 \leftarrow 0$ and $K_{a}=1 \leftarrow 0$. Several new higher $J$ transitions were measured due to a new OROTRON part, which enables detection in higher frequency ranges (130 $170 \mathrm{GHz}$ ). For ${ }^{20} \mathrm{Ne}-\mathrm{CO}$ and ${ }^{22} \mathrm{Ne}-\mathrm{CO}$ it was possible to combine these newly obtained data with the $a$-type transitions from [2], and with those measured in the IR [1]. To obtain rotational constants two approaches were chosen:

(i) The description of the complex as an asymmetric rotor by the aid of the Pickett software package, and

(ii) a model Hamiltonian for a near-symmetric rotor in conjunction with standard fit routines.

Throughout the paper, the $K$ quantum number is used in order to designate the $K_{a}$ of $\mathrm{Ne}-\mathrm{CO}$ in the asymmetric rotor notation.

\section{Experimental Details}

The experimental data presented in this paper were obtained with a newly designed and constructed 


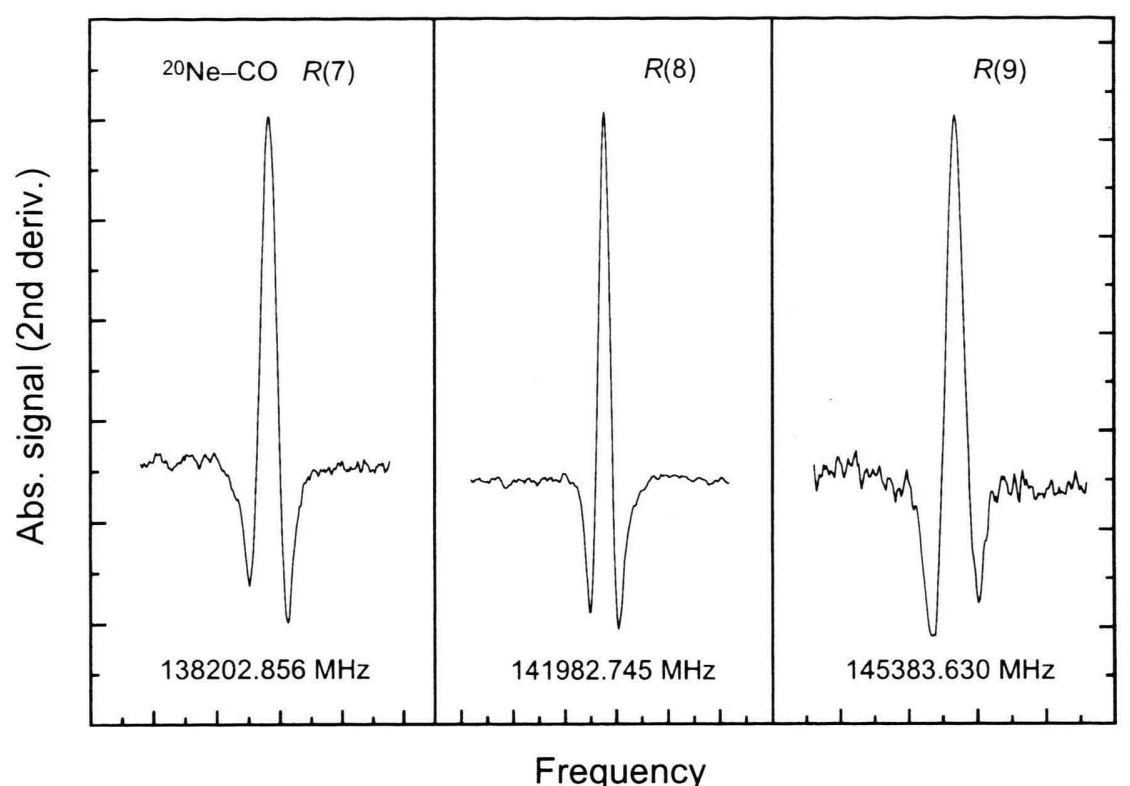

Fig. 1. Recording of three $R-$ branch transitions of the main isotopomer ${ }^{20} \mathrm{Ne}-\mathrm{CO}$ with the intracavity OROTRON spectrometer.
Table 1. $R$-branch transitions of ${ }^{20} \mathrm{Ne}-\mathrm{CO}(v=0 \leftarrow 0, K=$ $1 \leftarrow 0$ ) measured with the orotron spectrometer (in $\mathrm{MHz}$ ).

\begin{tabular}{lcrr}
\hline & OBS & O-C & \multicolumn{1}{c}{$\mathrm{O}-\mathrm{C}^{\mathrm{b}}$} \\
\hline$R(1)$ & $108640.311(30)$ & -0.032 & -0.026 \\
$R(2)$ & $114328.691(30)$ & -0.024 & -0.013 \\
$R(3)$ & $119724.459(30)$ & -0.002 & 0.008 \\
$R(4)$ & $124819.602(30)$ & 0.046 & 0.040 \\
$R(5)$ & $129605.197(30)$ & -0.011 & -0.030 \\
$R(6)$ & $134070.967(50)$ & 0.042 & 0.066 \\
$R(7)$ & $138202.856(100)$ & -0.231 & -0.083 \\
$R(8)$ & $141982.745(100)$ & -0.128 & 0.088 \\
$R(9)$ & $145383.630(100)$ & 0.218 & -0.025 \\
$R(10)$ & $148366.100(100)$ & -0.069 & $\mathrm{c}$ \\
\hline
\end{tabular}

a Values refer to the asymmetric rotor fit (i);

$\mathrm{b}$ values refer to the near-symmetric rotor fit (ii);

c omitted from near-symmetric rotor fit.

highly sensitive intracavity millimeter-wave spectrometer called OROTRON, in combination with a pulsed supersonic jet.

The cavity consists of a semi-concentric FabryPérot arrangement with a movable spherical mirror and a fixed planar mirror. This system serves as a high-quality resonant cavity and simultaneously as a source for the generation of millimeter waves [4].

The radiation is generated due to the planar mirror's periodic structure, which modulates the velocity of an electron beam emitted by a heated cathode. Those frequencies are amplified, which correspond to one of the cavity modes. The cavity is also used as the probe cell for the gases to be investigated. Therefore the cavity consists of two parts: a high vacuum region with the electron beam, which is separated from the pulsed-jet compartment by a mica-window. Absorption due to molecules in the jet is directly affecting the current of electrons which reach the collector.

The radiation frequency is measured by coupling out a small fraction of the power and mixing it with the output of a microwave synthesizer. The difference signal is then counted by a frequency meter. Signal to noise ratio enhancement is achieved by a double modulation technique - the $25 \mathrm{kHz}$ source modulation of the OROTRON and the $5-10 \mathrm{~Hz}$ jet modulation are demodulated by a lock-in amplifier and a gated integrator.

The OROTRON was mounted inside a vacuum chamber. The supersonic jet was injected into the cavity by a pulsed valve and pumped out of the vacuum chamber by a 1000 1/s diffusion pump. Typically, $2 \%$ of $\mathrm{CO}$ in $\mathrm{Ne}$ was used for the production of the $\mathrm{Ne}-\mathrm{CO}$ complexes. All measurements with ${ }^{22} \mathrm{Ne}-\mathrm{CO}$ were performed in natural abundance. The preliminary frequency predictions for ${ }^{20} \mathrm{Ne}-\mathrm{CO}$ and ${ }^{22} \mathrm{Ne}-\mathrm{CO}$ were calculated on the basis of the IR work [1].

\section{Analysis and Discussion}

The frequencies of the newly measured transitions of the $\mathrm{Ne}-\mathrm{CO}$ isotopomers are given in Tables 1 and 2 . To give an impression of how the signal quality looks like, three $R$-branch transitions with higher $J$ for 


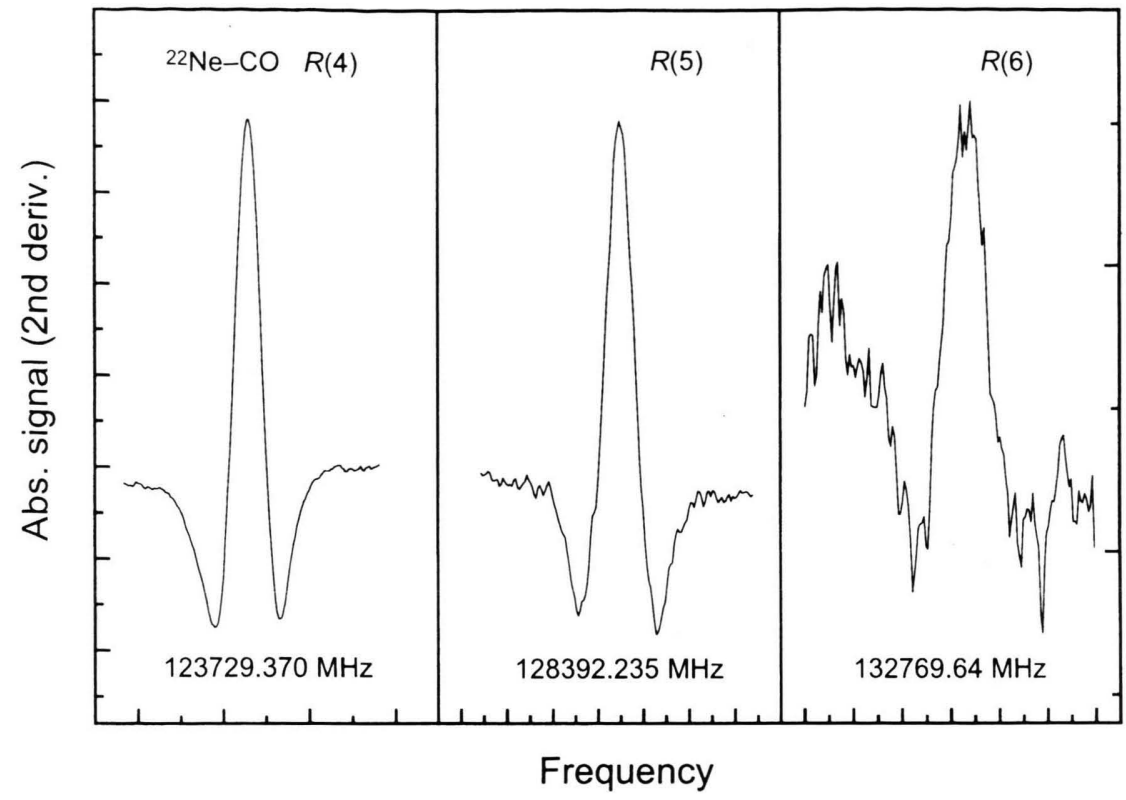

Fig. 2. OROTRON recording of $R(4), R(5)$, and $R(6)$ for ${ }^{22} \mathrm{Ne}-\mathrm{CO}$ (relative abundance is $8.82 \%$ ).
Table 2. $R$-branch transitions of ${ }^{22} \mathrm{Ne}-\mathrm{CO}(v=0 \leftarrow 0, K=$ $1 \leftarrow 0$ ) measured with the orotron spectrometer (in $\mathrm{MHz}$ ).

\begin{tabular}{llcr}
\hline & \multicolumn{1}{c}{ OBS } & O-C & O- $\mathrm{C}^{\mathrm{b}}$ \\
\hline$R(1)$ & $108123.275(30)$ & -0.026 & -0.007 \\
$R(2)$ & $113586.458(30)$ & 0.053 & 0.019 \\
$R(3)$ & $118791.080(50)$ & -0.061 & -0.054 \\
$R(4)$ & $123729.370(50)$ & -0.039 & 0.022 \\
$R(5)$ & $128392.235(50)$ & 0.019 & -0.006 \\
$R(6)$ & $132769.64(30)$ & 0.49 & 0.28 \\
$R(7)$ & $136847.1(5)$ & -0.7 & -0.4 \\
\hline
\end{tabular}

a Values refer to the asymmetric rotor fit (i);

$\mathrm{b}$ values refer to the near-symmetric rotor fit (ii).

each of the two isotopomers ${ }^{20} \mathrm{Ne}-\mathrm{CO}$ and ${ }^{22} \mathrm{Ne}-$ $\mathrm{CO}(8.82 \%$ natural abundance) are shown in Figs 1 and 2 .

The $\mathrm{Ne}-\mathrm{CO}$ transitions measured during this work were fitted together with the microwave data [2] and the IR data [1]. This was carried out in two ways: (i) By treating $\mathrm{Ne}-\mathrm{CO}$ as an asymmetric rotor. For this model fitting, the widely utilized software package by Pickett [9] was used (only with ground state data); (ii) a near-symmetric rotor energy level expression [7, 8] was used in fitting the different $K$-branches individually.

(i) In the case of the asymmetric rotor model for ${ }^{20} \mathrm{Ne}-\mathrm{CO}$, four $a$-type rotational transitions with $K=0$, five $a$-type rotational transitions with $K=1$ [2], and ten $b$-type rotational transitions between $K=0$ and $K=1$ of the present work were included
Table 3. Rotational constants for ${ }^{20} \mathrm{Ne}-\mathrm{CO}$ and ${ }^{22} \mathrm{Ne}-\mathrm{CO}$ treated as an asymmetric rotor (the values $A$ are shifted upwards by the IR values of $D_{K}, 0.2485 \mathrm{~cm}^{-1}$ and $0.2461 \mathrm{~cm}^{-1}$ respectively [1]).

\begin{tabular}{lccc}
\hline & $\begin{array}{c}{ }^{20} \mathrm{Ne}-\mathrm{CO} \\
\text { this work }\end{array}$ & $\begin{array}{c}{ }^{20} \mathrm{Ne}-\mathrm{CO} \\
\text { Walker } \text { et al. }\end{array}$ & $\begin{array}{c}{ }^{22} \mathrm{Ne}-\mathrm{CO} \\
\text { this work }\end{array}$ \\
\hline$A / \mathrm{MHz}$ & $107127.021(14)$ & $107000^{\mathrm{a}}$ & $106927.307(78)$ \\
$B / \mathrm{MHz}$ & $3479.6597(95)$ & $3479.99308(99)$ & $3298.2(21)$ \\
$C / \mathrm{MHz}$ & $3039.5387(93)$ & $3039.22634(80)$ & $2895.1(21)$ \\
$\Delta_{J} / \mathrm{kHz}$ & $322.822(38)$ & $327.613(40)$ & $291.2(11)$ \\
$\Delta_{J K} / \mathrm{kHz}$ & $24074.1(52)$ & $23916.24(35)$ & $16900(1000)$ \\
$\delta_{J} / \mathrm{kHz}$ & $-20.55(89)$ & $9.282(53)$ & $196.7(78)$ \\
$\Phi_{J} / \mathrm{kHz}$ & $-0.1380(25)$ & $-0.1225(13)$ & $-0.2923(73)$ \\
$\Phi_{J K} / \mathrm{kHz}$ & $3.35(94)$ & $-22.560(23)$ & $-217.9(72)$ \\
$\phi_{J} / \mathrm{kHz}$ & $-1.336(45)$ & $0.1530(21)$ & \\
$L_{J} / \mathrm{Hz}$ & $-0.225(45)$ & & \\
$L_{J J K} / \mathrm{Hz}$ & $-1463(46)$ & & \\
\hline
\end{tabular}

${ }^{a}$ Fixed to the value obtained from the IR data.

in the fit file for the Pickett software package. The line positions are reproduced by the fit with experimental accuracy. The constants, which are shown in Table 3, can be compared with those given in [2]. For reference, these constants are given in the third column of Table 3. The observation of the $b$-type millimeter wave transitions allowed us to determine accurately the value of the difference $A-D_{K}$ directly from the fit. The $D_{K}$ centrifugal distortion constant was fixed on the value of $0.2485 \mathrm{~cm}^{-1}$, which was obtained in the IR work [1]. The corresponding value of $A$ is given in Table 3. 
For ${ }^{22} \mathrm{Ne}-\mathrm{CO}$, only four $a$-type rotational transitions with $K=0$ were detected in the microwave range [2]. As the recorded seven $b$-type $R$-branch transitions of the present study probe only one of the two asymmetry splitting components of the $K=1$ state, additional information was necessary for the fit in order to determine the splitting parameters. Such information could be obtained in the millimeter wave range by measuring the $Q$-branch of $K=1 \leftarrow 0 b$ type transitions, which probe another splitting component. These transitions are presently outside the spectral range of our intracavity OROTRON jet spectrometer, and therefore they could not be measured in this work. The appropriate microwave data concerning the $a$-type transitions in the $K=1$ state were also absent for ${ }^{22} \mathrm{Ne}-\mathrm{CO}$.

Instead, we obtained the transition frequencies of two $Q$-branch $b$-type millimeter wave transitions by combining the available infrared and microwave data given in [1] and [2]. These two transitions with their "experimental" frequencies, namely $Q(2)$ at $96936 \mathrm{MHz}$ and $Q(3)$ at $97466 \mathrm{MHz}$, were included in the fit with appropriately lower weights (according to the IR accuracy of $30 \mathrm{MHz}$ ). The resulting constants are listed in the last column of Table 3. Similarly to the ${ }^{20} \mathrm{Ne}-\mathrm{CO}, A-D_{K}$ was obtained directly from the fit and the given $A$ value is shifted upwards by the IR value of $D_{K}=0.2461 \mathrm{~cm}^{-1}$.

(ii) The second approach for retrieving molecular parameters was composed of interpreting $\mathrm{Ne}-\mathrm{CO}$ as a near-symmetric rotor. The following energy level expression was already successfully used for $\mathrm{Ar}-\mathrm{CO}$ and $\mathrm{Kr}-\mathrm{CO}$. In [1] it was employed to fit about 105 transitions for ${ }^{20} \mathrm{Ne}-\mathrm{CO}$ and 88 for ${ }^{22} \mathrm{Ne}-\mathrm{CO}$, which were assigned to perpendicular subbands with $K=$ $1 \leftarrow 0$ and $K=0 \leftarrow 1$ and a parallel subband with $K=0 \leftarrow 0$.

$E_{\text {total }}=\sigma+\bar{B}\left[J(J+1)-K^{2}\right]-D\left[J(J+1)-K^{2}\right]^{2}$ $+H\left[J(J+1)-K^{2}\right]^{3}+L\left[J(J+1)-K^{2}\right]^{4}+\Delta E_{K}$,

$\Delta E_{K}= \pm(1 / 2)\{(1 / 2)(B-C) J(J+1)$

$$
\left.+d[J(J+1)]^{2}+h[J(J+1)]^{3}\right\} .
$$

To reproduce the measured transitions within experimental accuracy and to have the parameters well defined we added $L$ and $h$ for the $v(\mathrm{CO})=0$ states. The $\sigma$ are the band origins of each stack of levels
Table 4. Molecular parameters of ${ }^{20} \mathrm{Ne}-\mathrm{CO}\left(\right.$ in $\left.\mathrm{cm}^{-1}\right)$.

\begin{tabular}{cccc}
\hline & & $v(\mathrm{CO})=1$ & \multicolumn{1}{c}{$v(\mathrm{CO})=0$} \\
\hline$K=0$ & $\sigma$ & $2143.200061(76)$ & 0.0 \\
& $\bar{B}$ & $0.1085984(58)$ & $0.108732742(29)$ \\
& $D$ & $0.13036(86) \times 10^{-4}$ & $0.128731(32) \times 10^{-4}$ \\
& $H$ & $-0.401(30) \times 10^{-8}$ & $-0.457(11) \times 10^{-8}$ \\
& $L$ & $0.0^{\mathrm{a}}$ & $0.94(51) \times 10^{-12}$ \\
$K=1$ & $\sigma$ & $2146.494384(84)$ & $3.32406009(50)$ \\
& $\bar{B}$ & $0.1077444(61)$ & $0.107910654(25)$ \\
& $D$ & $0.1132(10) \times 10^{-4}$ & $0.121684(42) \times 10^{-4}$ \\
& $H$ & $-0.1200(46) \times 10^{-7}$ & $-0.492(23) \times 10^{-8}$ \\
& $L$ & $0.0^{\mathrm{a}}$ & $-0.1896(37) \times 10^{-10}$ \\
& $B-C$ & $0.0147140(82)$ & $0.01470113(13)$ \\
& $d$ & $0.473(38) \times 10^{-6}$ & $-0.5163(94) \times 10^{-6}$ \\
& $h$ & $0.0^{\mathrm{a}}$ & $0.551(45) \times 10^{-8}$ \\
\hline
\end{tabular}

a $L$ and $h$ not introduced for $v(\mathrm{CO})=1$ levels.

Table 5. Molecular parameters of ${ }^{22} \mathrm{Ne}-\mathrm{CO}\left(\right.$ in $\left.^{-1}\right)$.

\begin{tabular}{cccl}
\hline & & $v(\mathrm{CO})=1$ & \multicolumn{1}{c}{$v(\mathrm{CO})=0$} \\
\hline$K=0$ & $\sigma$ & $2143.20018(22)$ & 0.0 \\
& $\bar{B}$ & $0.1031223(60)$ & $0.103295109(26)$ \\
& $D$ & $0.11066(45) \times 10^{-4}$ & $0.114808(28) \times 10^{-4}$ \\
& $H$ & $-0.5074(84) \times 10^{-8}$ & $-0.3364(93) \times 10^{-8}$ \\
& $L$ & $0.0^{\mathrm{a}}$ & $-0.214(24) \times 10^{-11}$ \\
& & & \\
& $\sigma$ & $2146.49120(46)$ & $3.3199590(59)$ \\
& $B$ & $0.102403(18)$ & $0.1026416(54)$ \\
& $D$ & $0.898(20) \times 10^{-5}$ & $0.10805(65) \times 10^{-4}$ \\
& $H$ & $-0.1511(64) \times 10^{-7}$ & $-0.555(36) \times 10^{-8}$ \\
& $L$ & $0.0^{\mathrm{a}}$ & $-0.195(16) \times 10^{-10}$ \\
& $B-C$ & $0.013186(17)$ & $0.013160(22)$ \\
& $d$ & $0.732(66) \times 10^{-6}$ & $0.40(12) \times 10^{-6}$ \\
\hline
\end{tabular}

${ }^{a} L$ not introduced for $v(\mathrm{CO})=1$ levels

denoted by $v_{\mathrm{CO}}$ and $K$. The asymmetry splitting term, $\Delta E_{K}$, is zero for $K=0$.

In case of the main isotopic modification ${ }^{20} \mathrm{Ne}-$ $\mathrm{CO}$, it was shown in [3] that the IR experimental data concerning higher $K$ states of the complex are better described by representing each individual stack of asymmetry-doubling levels with an independent power series and by keeping only the common band origin. Such a global fit of all the infrared and microwave data was carried out for ${ }^{20} \mathrm{Ne}-\mathrm{CO}$ in [3]. Here we confined ourselves on the incorporation of the present millimeter wave data ( 9 transitions), the microwave data ( 9 transitions) and the data from the infrared concerning the states with $K=0$ and $K=1$. The results of the fit are given in Table 4 .

In the case of ${ }^{22} \mathrm{Ne}-\mathrm{CO}$, the 7 transitions measured with the OROTRON were fitted together with $4 a$ - 
type microwave transitions and the data from the IR. The results of the fit are given in Table 5 .

The molecular parameters obtained for ${ }^{20} \mathrm{Ne}-\mathrm{CO}$ and ${ }^{22} \mathrm{Ne}-\mathrm{CO}$ are in good agreement with those given by Randall et al. [1]. We note here, that the millimeter wave and the microwave transitions were measured with much higher accuracy than the IR data. Therefore their use allowed us to determine the constants of the fit with higher accuracy in comparison with [1]. In particular, the band origin for the $K=1$ stack of the complex could be obtained now very accurately.

In both fitting models the number of parameters was chosen that way to have $\mathrm{O}-\mathrm{C}$ values within or near the experimental accuracy and to have the parameters well defined. Therefore e.g. in the case of the near-symmetric rotor the parameters $L$ and $h$ were not introduced for the $v(\mathrm{CO})=1$ levels because we have sufficient precise measurements only for the ground state. One can compare $\mathrm{O}-\mathrm{C}$ values for both models in the Tables 1 and 2 . Both models, the near-symmetric rotor and the asymmetric rotor, show their limitations in the case of $\mathrm{Ne}-\mathrm{CO}$, which is considerably more unrigid than similar complexes like $\mathrm{Ar}-\mathrm{CO}$ or $\mathrm{Kr}-\mathrm{CO}$. In the case of the asymmetric rotor e.g. $\Phi_{J K}$ and the parameter $L_{J J K}$, which is of next higher order, are of comparable absolute value. In the near-symmetric rotor fit we omitted the $R(10)$ transition of ${ }^{20} \mathrm{Ne}-\mathrm{CO}$ in the fit because $\mathrm{O}-\mathrm{C}$ values went too high otherwise. Adding an additional constant to gain better results leads to less well defined constants.

[1] R. W. Randall, A. J. Cliffe, B. J. Howard, and A. R. W. McKellar, Mol. Physics 79, 1113 (1993).

[2] K. A. Walker, T. O. Ogata, W. Jäger, M. C. L. Gerry, and I. Ozier, J. Chem. Phys. 106, 7519 (1997).

[3] A. R. W. McKellar and M.-C. Chan, Mol. Physics 93, 253 (1998).

[4] G. Winnewisser, B. S. Dumesh, I. Pak, L. A. Surin, F. Lewen, D. A. Roth, and F. S. Rusin, J. Mol. Spectrosc. 192, 243 (1998).

[5] R. Moszynski, T. Korona, P.E. S. Wormer, and A. van der Avoird, J. Phys. Chem. A 101, 4690 (1997).
In comparison to the $\mathrm{Ar}-\mathrm{CO}$ complex, which was first observed by DePiante et al. [10], much less data are available for the $\mathrm{Ne}-\mathrm{CO}$ complex. Especially, the ${ }^{22} \mathrm{Ne}-\mathrm{CO}$ and ${ }^{21} \mathrm{Ne}-\mathrm{CO}$ complexes are still not well characterized. The situation could be substantially improved by millimeter wave observations of the $Q-$ branch $K_{a}=1 \leftarrow 0 b$-type rotational transitions. This would make possible the first determination of the rotational constants of the ${ }^{21} \mathrm{Ne}-\mathrm{CO}$ complex, and would allow an improvement of the accuracy of the rotational constants of ${ }^{22} \mathrm{Ne}-\mathrm{CO}$.

\section{Acknowledgements}

This work was supported in part by the Deutsche Forschungsgemeinschaft (DFG) via Grant SFB 494 and by special grants from the Ministry of Science and Technology of the Land Nordrhein-Westfalen. The support of the Institute of Spectroscopy at Troitsk through grants of the Russian Foundation for $\mathrm{Ba}-$ sic Research (RFBR: 00-02-17525) is gratefully acknowledged by L.A.S. and B.S.D. The work of the Russian colleagues, I.P., L.A.S., and B.S.D., at Cologne was made possible by the DFG through a grant aimed at supporting Eastern and Central European Countries and the Republics of the former Soviet Union. The authors wish to thank Holger S. P. Müller for his assistance in the operation of the Pickett software package and Frank Lewen for technical assistance.

[6] G.C. McBane and S. M. Cybulski, J. Chem. Phys. 110, 11734 (1999).

[7] A. R. W. McKellar, Y. P. Zeng, S. W. Sharpe, C. Wittig, and R. A. Beaudet, J. Mol. Spectrosc. 153, 475 (1992).

[8] A.R.W. McKellar, J. Mol. Spectrosc. 158, 100 (1993).

[9] H. M. Pickett, J. Mol. Spectrosc. 148, 371 (1991).

[10] A. DePiante, E. J. Campbell, and S. J. Buelow, Rev. Sci. Instrum. 60, 858 (1989). 